\title{
Fuzzy Membership Function Dependent Switched Control for Nonlinear Systems with Memory Sampled-Data Information
}

\section{B. Visakamoorthi}

The Gandhigram Rural Institute Deemed University Department of Mathematics

\section{K. Subramanian}

Kunsan National University College of Engineering

Muthukumar Palanisamy ( $\nabla$ pmuthukumargri@gmail.com )

The Gandhigram Rural Institute Deemed University Department of Mathematics https://orcid.org/0000-0003-1396-9948

\section{Research Article}

Keywords: T-S fuzzy systems, Lyapunov-Krasovskii functional, memory sampled-data control, Linear matrix inequality, generalized $\mathrm{N}$-order free-matrix-based inequality.

Posted Date: November 17th, 2021

DOI: https://doi.org/10.21203/rs.3.rs-1008996/v1

License: (9) (1) This work is licensed under a Creative Commons Attribution 4.0 International License. Read Full License 


\title{
Fuzzy membership function dependent switched control for nonlinear systems with memory sampled-data information
}

\author{
B. Visakamoorthi ${ }^{a}$, K. Subramanian ${ }^{b}$, P. Muthukumar ${ }^{d 1}$. \\ ${ }^{a}$ Department of Mathematics, The Gandhigram Rural Institute (Deemed to be University), \\ Gandhigram - 624 302, Tamil Nadu, India. \\ ${ }^{b}$ School of IT Information and Control Engineering, Kunsan National University, 558, \\ Daehak-ro, Gunsan-si, Jeollabuk-do, 54150, Republic of Korea.
}

\begin{abstract}
In this paper, a fuzzy memory-based coupling sampled-data control (SDC) is designed for nonlinear systems through the switched approach. Compared with the usual SDC scheme, by employing the Bernoulli sequence, a more general coupling switched SDC that involving the signal transmission delay is designed. The LyapunovKrasovskii Functional (LKF) is presented with the available characteristics of the membership function, and a coupling sampling pattern, for the T-S fuzzy systems. Based on LKF, together with time derivative information of membership function, and the generalized $N$-order free-matrix-based inequality, the suitable conditions are obtained in terms of linear matrix inequalities (LMIs) for guaranteeing the asymptotic stability and stabilization of the concerned system. Then the desired fuzzy coupling SDC gain is attained from the solvable LMIs. In the end, two examples are given to validate the derived theoretical results.
\end{abstract}

Keywords: T-S fuzzy systems; Lyapunov-Krasovskii functional; memory sampled-data control; Linear matrix inequality; generalized $N$-order free-matrix-based inequality.

\section{INTRODUCTION}

The analysis and synthesis of nonlinear dynamical systems have gradually become the focus of attention due to extensive applications in physics, engineering communities, and so on 112 . In real life, stability analysis and control design for nonlinear dynamical systems are quite difficult. Recently, the T-S fuzzy model has been dealt with the intrinsic nonlinear systems because the T-S fuzzy model can

\footnotetext{
${ }^{1}$ Corresponding Author; Email: pmuthukumargri@gmail.com, Phone: 91-451-2452371, Fax: 91-451-2454466. visakamoorthi8596@gmail.com (B. Visakamoorthi), subramaniangri@gmail.com (K. Subramanian).
} 
depict the considered nonlinear systems as a weighted sum of some linear subsystems. From this idea, the stabilization for T-S fuzzy systems has been established in 3 7. Moreover, the various nonlinear dynamical systems such as Rossler's system [2], Chua's circuit [8], mass-spring system [9], have been formulated as T-S fuzzy systems to study the stabilization problem. To tackle the stabilization issue of T-S fuzzy systems, several control techniques have been designed in literature, for example, adaptive control [10], impulsive control [11, mixed $H_{\infty}$ passive control [12, SDC [13, and so on.

With the rapid development of communication technology and digital networks, SDC becomes an attractive field of research in the control system. The main scope of the SDC is updating the control signal information only at the sampling instants, not for the whole time interval, which reduces the communication bandwidth [14. Also, the SDC has many advantages when compared with continuoustime controllers, such as efficiency, maintenance with low cost, and simple installation. According to these applications, the SDC has been utilized to investigate the various problems in T-S fuzzy systems 14 18. For instance, the dissipativity and extended dissipativity for T-S fuzzy systems have been investigated via SDC in $[19$ and 20 , respectively. Moreover, the asymptotic stabilization of T-S fuzzy-based chaotic systems has been studied via the SDC scheme in [21].

Further, the updating signal successfully transmitted from sampler to the controller and to the zero-order-hold $(\mathrm{ZOH})$, the control signal may experience the constant transmission delay at any time instant $t_{k}$, which leads to the necessity of the memory-based SDC 22 . According to this viewpoint, the memory-based SDC has been designed for the stabilization of T-S fuzzy systems in [22,23]. Also, the dissipativity of T-S fuzzy systems has been analyzed via memory SDC in 24. Very recently, the stabilization problem has been studied via memory SDC for T-S fuzzy time-delay system. From the above literature, memory SDC has improved the stability performance of the proposed systems with less conservative results. Inspired from the above, the memory-based coupling SDC which combines both the traditional SDC and memory-based SDC with the help of the Bernoulli sequence, is designed to improve the asymptotic stability of T-S fuzzy systems in the present study.

Meanwhile, when investigating the stabilization of T-S fuzzy systems under SDC scheme, the less conservative results through the choosing of proper sampling interval is significant. Since, the maximum sampling interval gives some superiorities, like lower communication channel occupation, less signal transmission, and less actuation of the controller. Hence, several methods have been established in the literature 25,28 to get the less conservatism via largest upper bounds (LUB) 
Fuzzy membership function dependent switched control for nonlinear systems with memory sampled-data information 3

of sampling interval which ensures the stabilization of T-S fuzzy systems. In particular, a loopedfunctional has been introduced in [29], which relax the positivity condition of LKF and also obtained the less conservative results. Nowadays, a new two-sided looped-functional has been employed to improve the stability condition from the information of the whole sampling interval and greatly enlarge the maximum sampling intervals 30,31 .

Moreover, in fuzzy systems, membership functions play a crucial role to analyze the stability of systems and acquire the less conservatism. So, the time derivative of fuzzy membership function dependent LKFs has been employed for the stability analysis of T-S fuzzy systems in [32]. For example, the stability problem has been studied for T-S fuzzy systems via membership function dependent LKF in [33. Furthermore, when compared with usual control method [13], switched control approach for T-S fuzzy systems diminish the number of LMIs and attain maximum sampling interval, which has been studied in 33 34]. Although a lot of attempts had made on the investigation of T-S fuzzy systems stability, when the memory-based coupling switched SDC information is taken into account, the stability of fuzzy systems has been drawn very little research, which motivates us to carry on the current research. Hence, this paper aims to propose SDC strategy for T-S fuzzy systems together with the coupling sampling pattern, membership function dependent LKF, and applying the switching topology. Through this, we attempt to ensure better performance for T-S fuzzy systems when compared to the existing works with the complete information about the time derivative of chosen membership function.

Inspired from the above, the stabilization of T-S fuzzy systems is investigated in this paper via memory-based coupling SDC. The main contributions of the paper lie in the following aspects:

(1) Different from the conventional SDC and memory-based SDC scheme $13,14,23,24$ a more general memory-based coupling SDC is designed by Bernoulli sequence for the T-S fuzzy systems.

(2) Unlike from the LKF $14,19,21,26$, the membership function dependent LKF, which including the available information of the coupling sampling pattern, signal transmission delay, and full state information from $t$ to $t_{k}$ and $t$ to $t_{k+1}$ is constructed in the present study.

(3) By utilizing the generalized $N$-order free-matrix-based inequality, the stabilization criteria are derived with the aid of time derivative of membership function via switched approach for the considered systems in the form of LMIs. 
(4) To show the effectiveness of designed control method, the derived stabilization conditions are compared with existing works $13,14,28$, and the designed controller achieves the LUB of sampling interval with less conservative results.

The paper has the following structure: Section 2 devotes the formulation of T-S fuzzy system. In Section 3, the memory-based coupling SDC is designed for T-S fuzzy system. Two examples are considered in Section 4. Conclusions are drawn in Section 5.

Notations: $\mathbb{R}^{n \times m}$ and $\mathbb{R}^{n}$ denote $n \times m$ real matrix and the $n$-dimensional Euclidean space, respectively. The matrix $U>0(<0)$ denotes a positive (negative) definite. $\mathbb{E}\{\cdot\}$ indicates the mathematical expectation operator. $\operatorname{Sym}\{A\}=A+A^{T}$. I and 0 represent the identity and zero matrix with appropriate dimensions, respectively. $\operatorname{diag}\{\cdots\}$ is a block diagonal matrix.

\section{Preliminaries and System Description}

2.1. T-S Fuzzy System Formulation: Let us consider the nonlinear systems as follows:

$$
\dot{x}(t)=h(x(t), u(t)),
$$

where $x(t) \in \mathbb{R}^{n}$ and $u(t) \in \mathbb{R}^{m}$ represent the state and control input vector, respectively. $h(x(t), u(t))$ denotes a known nonlinear function which satisfies $h(0,0)=0$. Then, based on the T-S fuzzy modeling approach, nonlinear system (1) can be represented by a series of IF-THEN rules:

Rule $i$ : IF $w_{1}(t)$ is $\theta_{1}^{i}, w_{2}(t)$ is $\theta_{2}^{i}, \cdots, w_{p}(t)$ is $\theta_{p}^{i}$, THEN,

$$
\dot{x}(t)=A_{i} x(t)+B_{i} u(t), \quad i \in \Delta=\{1,2, \cdots, r\},
$$

where $A_{i} \in \mathbb{R}^{n \times n}$ and $B_{i} \in \mathbb{R}^{n \times m}$ are constant matrices; $w_{1}(t), w_{2}(t), \cdots, w_{p}(t)$ are the premise variables; $\theta_{1}^{i}, \theta_{2}^{i}, \cdots, \theta_{p}^{i}$ denote the fuzzy sets.

By utilizing product inference, singleton fuzzifier and center average defuzzifier, the whole T-S fuzzy system (2) can be inferred as

$$
\dot{x}(t)=\sum_{i=1}^{r} \delta_{i}(w(t))\left[A_{i} x(t)+B_{i} u(t)\right]
$$

where $w(t)=\left[w_{1}(t), w_{2}(t), \cdots, w_{p}(t)\right]^{T}, \delta_{i}(w(t))$ is the normalized membership function satisfying $\delta_{i}(w(t))=\frac{\prod_{g=1}^{p} \theta_{g}^{i}\left(w_{g}(t)\right)}{\sum_{i=1}^{r} \prod_{g=1}^{p} \theta_{g}^{i}\left(w_{g}(t)\right)} \geq 0$, and $\sum_{i=1}^{r} \delta_{i}(w(t))=1$ and $\theta_{g}^{i}\left(w_{g}(t)\right)$ is the grade of membership of $w_{g}(t)$ in $\theta_{g}^{i}$. 
Fuzzy membership function dependent switched control for nonlinear systems with memory sampled-data information 5

2.2. Design of Memory-based Coupling SDC Strategy. In this subsection, the memory-based coupling SDC is designed for the system (3). For this, the control input signal is assumed to be generated by utilizing a ZOH function with $0=t_{0}<t_{1}<\cdots<t_{k}<\cdots, \lim _{k \rightarrow \infty} t_{k}=+\infty$.

Rule $j$ : IF $w_{1}(t)$ is $\theta_{1}^{j}, w_{2}(t)$ is $\theta_{2}^{j}, \cdots, w_{p}(t)$ is $\theta_{p}^{j}$, THEN,

$$
u(t)=\beta(t) K_{j} x\left(t_{k}-\tau\right)+(1-\beta(t)) L_{j} x\left(t_{k}\right), \quad t \in\left[t_{k}, t_{k+1}\right), \quad j \in \Delta
$$

where $K_{j}$ and $L_{j}$ are the control gain matrices and $\tau$ is constant signal transmission delay. Now, define $d(t)=t-t_{k}$ with $\dot{d}(t)=1$ for $t \neq t_{k}$ and which satisfies $0<d(t) \leq d_{k}=t_{k+1}-t_{k} \leq \bar{d}, \bar{d}$ is the maximum sampling interval. Here $\beta(t)$ is the Bernoulli stochastic variable coupling the conventional SDC and memory-based SDC with $\operatorname{Pr}\{\beta(t)=1\}=\mathbb{E}\{\beta(t)\}=\beta$ and $\operatorname{Pr}\{\beta(t)=0\}=1-\mathbb{E}\{\beta(t)\}=1-\beta$ where $\beta \in[0,1]$. Then, the overall memory-based coupling SDC is represented as

$$
u(t)=\sum_{j=1}^{r} \delta_{j}\left(w\left(t_{k}\right)\right)\left[\beta(t) K_{j} x\left(t_{k}-\tau\right)+(1-\beta(t)) L_{j} x\left(t_{k}\right)\right], \quad t \in\left[t_{k}, t_{k+1}\right) .
$$

Based on the control input (4) and system (3), we get the fuzzy system as follows:

$$
\dot{x}(t)=\sum_{i=1}^{r} \sum_{j=1}^{r} \delta_{i}(w(t)) \delta_{j}\left(w\left(t_{k}\right)\right)\left[A_{i} x(t)+B_{i}\left(\beta(t) K_{j} x\left(t_{k}-\tau\right)+(1-\beta(t)) L_{j} x\left(t_{k}\right)\right)\right] .
$$

Remark 1. In the existing literature, the conventional SDC and memory-based SDC have been separately designed for T-S fuzzy systems. Distinct from this, the above-said controllers are coupled by the Bernoulli sequence in this present study. From the memory-based coupling SDC (4), when the stochastic variable $\beta(t)=0$, the designed control technique (4) reduces to the conventional $S D C$ input $u(t)=\sum_{j=1}^{r} \delta_{j}\left(w\left(t_{k}\right)\right) L_{j} x\left(t_{k}\right)$, which has been widely established for T-S fuzzy systems in [13, 14]. Meantime, if $\beta(t)=1$, then the designed controller becomes a memory-based SDC $u(t)=\sum_{j=1}^{r} \delta_{j}\left(w\left(t_{k}\right)\right) K_{j} x\left(t_{k}-\tau\right)$, which also has significantly designed for T-S fuzzy systems, recently (see, [23, 24]). From the above, the employed control technique for T-S fuzzy systems is more general than the works of $[13,14,23,24]$.

2.3. Switching Approach for Designing Control: Let us consider

$$
\begin{aligned}
& \dot{P}_{\delta}=\sum_{i=1}^{r} \dot{\delta}_{i}(w(t)) P_{i}=\sum_{j=1}^{r-1} \dot{\delta}_{j}(w(t))\left(P_{j}-P_{r}\right), \dot{Q}_{\delta}=\sum_{i=1}^{r} \dot{\delta}_{i}(w(t)) Q_{i}=\sum_{j=1}^{r-1} \dot{\delta}_{j}(w(t))\left(Q_{j}-Q_{r}\right), \\
& \dot{R}_{\delta}=\sum_{i=1}^{r} \dot{\delta}_{i}(w(t)) R_{i}=\sum_{j=1}^{r-1} \dot{\delta}_{j}(w(t))\left(R_{j}-R_{r}\right), \dot{S}_{\delta}=\sum_{i=1}^{r} \dot{\delta}_{i}(w(t)) S_{i}=\sum_{j=1}^{r-1} \dot{\delta}_{j}(w(t))\left(S_{j}-S_{r}\right),
\end{aligned}
$$




$$
\dot{U}_{\delta}=\sum_{i=1}^{r} \dot{\delta}_{i}(w(t)) U_{i}=\sum_{j=1}^{r-1} \dot{\delta}_{j}(w(t))\left(U_{j}-U_{r}\right), \dot{W}_{\delta}=\sum_{i=1}^{r} \dot{\delta}_{i}(w(t)) W_{i}=\sum_{j=1}^{r-1} \dot{\delta}_{j}(w(t))\left(W_{j}-W_{r}\right),
$$

where $P_{i}, S_{i}, U_{i}, Q_{i}, R_{i}, W_{i}$ are matrix variables to be obtained; and $\dot{\delta}_{i}(w(t))$ will be positive or negative which dependence upon of time. In order to ensure $\dot{P}_{\delta} \leq 0, \dot{Q}_{\delta} \leq 0, \dot{S}_{\delta} \leq 0, \dot{U}_{\delta} \leq 0, \dot{R}_{\delta} \leq 0$ and $\dot{W}_{\delta} \leq 0$, the following switching idea is utilized:

$$
\left\{\begin{array}{cl}
\text { IF } \dot{\delta}_{j}(w(t))<0, \text { THEN } & P_{j}-P_{r}>0, Q_{j}-Q_{r}>0, R_{j}-R_{r}>0, W_{j}-W_{r}>0, \\
& U_{j}-U_{r}>0, S_{j}-S_{r}>0 . \\
\text { IF } \dot{\delta}_{j}(w(t)) \geq 0, \text { THEN } & P_{j}-P_{r} \leq 0, Q_{j}-Q_{r} \leq 0, R_{j}-R_{r} \leq 0, W_{j}-W_{r} \leq 0 \\
& U_{j}-U_{r} \leq 0, S_{j}-S_{r} \leq 0 .
\end{array}\right.
$$

From (6), there are $2^{r-1}$ possible cases exist. Define $\mu \in \chi=\left\{1,2, \cdots, 2^{r-1}\right\}$, then, equation (6) can be expressed as

$$
\text { IF } \mathbb{A}_{\mu}, \text { THEN } \mathbb{B}_{\mu}
$$

where $\mathbb{A}_{\mu}$ and $\mathbb{B}_{\mu}$ represent the sets that contains the possible permutations of $\dot{\delta}(w(t))$ for $\mu \in \chi$ and all the possible constraints of $P_{i}, Q_{i}, R_{i}, W_{i}, U_{i}, S_{i}$ for $\mu \in \chi$, respectively. Based on the above, we get the following lemma as in 33 .

Lemma 1. Consider the system (3) with fuzzy membership function $\delta_{i}(w(t))$. For the symmetric matrices $P_{i}>0, U_{i}>0, S_{i}>0, Q_{i}, R_{i}$, and any matrix $W_{i}$, where $i \in \Delta$. If the switching rules (7) holds, then we have

$$
\dot{P}_{\delta} \leq 0, \dot{Q}_{\delta} \leq 0, \dot{S}_{\delta} \leq 0, \dot{U}_{\delta} \leq 0, \dot{R}_{\delta} \leq 0, \dot{W}_{\delta} \leq 0
$$

Now, from the Lemma 1 for different $\mathbb{A}_{\mu}$ and $\mathbb{B}_{\mu}$, we will design the corresponding coupling memorybased sampled-data switched control as follows:

$u_{\mu}=\beta(t) K_{\mu, \delta} x\left(t_{k}-\tau\right)+(1-\beta(t)) L_{\mu, \delta} x\left(t_{k}\right)$, where $K_{\mu, \delta}=\sum_{j=1}^{r} \delta_{j}\left(w\left(t_{k}\right)\right) K_{\mu, j}, L_{\mu, \delta}=\sum_{j=1}^{r} \delta_{j}\left(w\left(t_{k}\right)\right) L_{\mu, j}$. The final memory-based coupling sampled-data switched control (4) is expressed as

$$
u(t)=\left\{\begin{array}{c}
u_{1}=\beta(t) K_{1, \delta} x\left(t_{k}-\tau\right)+(1-\beta(t)) L_{1, \delta} x\left(t_{k}\right) \\
u_{2}=\beta(t) K_{2, \delta} x\left(t_{k}-\tau\right)+(1-\beta(t)) L_{2, \delta} x\left(t_{k}\right) \\
\vdots \\
u_{2^{r-1}}=\beta(t) K_{2^{r-1}, \delta} x\left(t_{k}-\tau\right)+(1-\beta(t)) L_{2^{r-1}, \delta} x\left(t_{k}\right) .
\end{array}\right.
$$




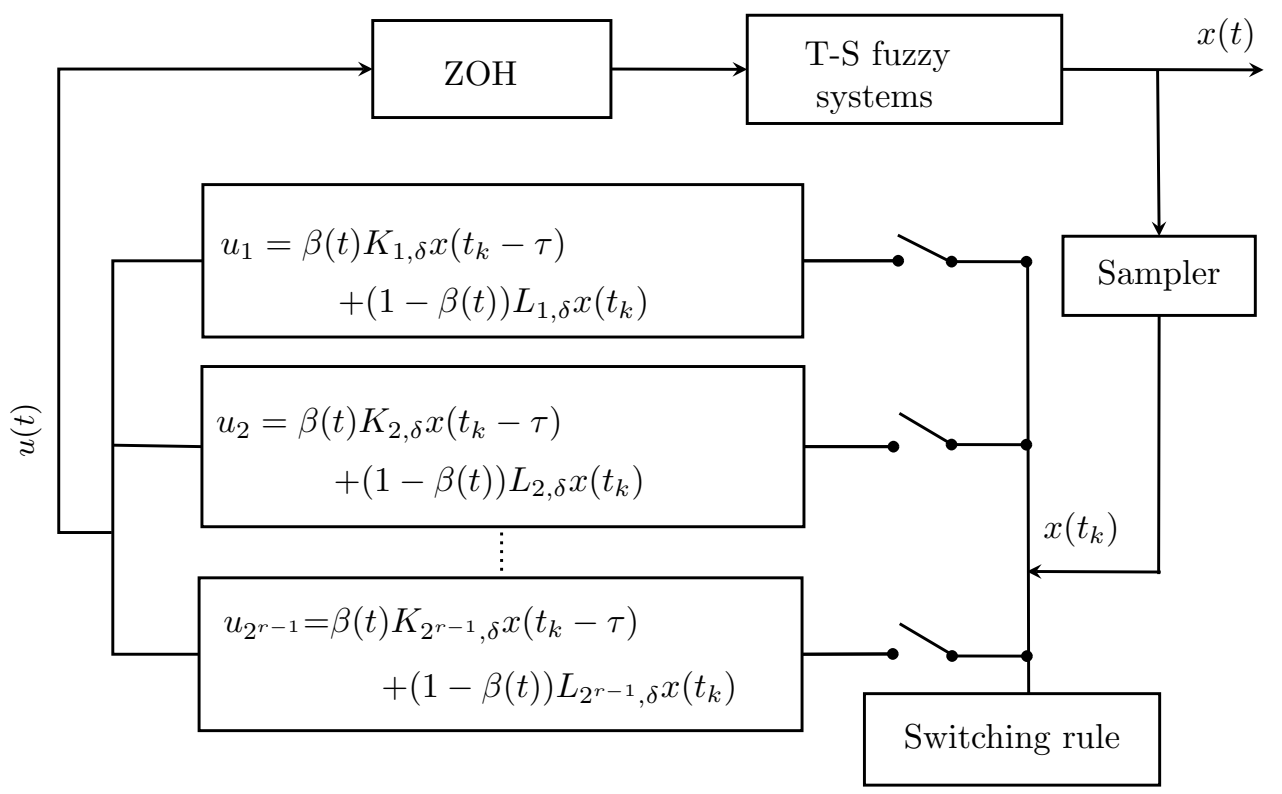

FIGURE 1. Block diagram of memory-based coupling sampled-data switched control system.

Meanwhile, the block diagram of the T-S fuzzy systems with memory-based coupling sampled-data switched control is presented in Figure 1. Hence, the closed-loop system with the control input (8) is given as follows:

$$
\dot{x}(t)=\sum_{i=1}^{r} \sum_{j=1}^{r} \delta_{i}(w(t)) \delta_{j}\left(w\left(t_{k}\right)\right)\left[A_{i} x(t)+B_{i}\left(\beta(t) K_{\mu, j} x\left(t_{k}-\tau\right)+(1-\beta(t)) L_{\mu, j} x\left(t_{k}\right)\right)\right] .
$$

Lemma 2. [Generalized $N$-order free-matrix-based inequality [35]] For scalars $c$ and $d(d>c), a$ vector $\beth_{N} \in \mathbb{R}^{m}(N \geq 0)$, the matrix $U>0 \in \mathbb{R}^{n \times n}$, any matrix $J=\left[J_{0}^{T}, J_{1}^{T}, \cdots, J_{N}^{T}\right]^{T}$ and a vector valued differentiable function $x:[c, d] \rightarrow \mathbb{R}^{n}$ such that

$$
-\int_{c}^{d} \dot{x}^{T}(s) U \dot{x}(s) d s \leq(d-c) \beth_{N}^{T} J^{T} \mathcal{R}_{N}^{-1} J \beth_{N}+\operatorname{Sym}\left\{\phi_{l N}^{T}(t) J^{T} \Pi_{l N} \phi_{l N}(t)\right\},
$$


holds. Here, $\Pi_{l N}=\Gamma_{l N} \Omega_{l N},(l=1,2), \quad \mathcal{R}_{N}=\operatorname{diag}\{U, 3 U, \cdots,(2 N+1) U\}$,

$$
\begin{aligned}
& \Gamma_{1 N}=\left[\begin{array}{cccc}
I & 0 & \cdots & 0 \\
I & (-1)^{1}\left(\begin{array}{c}
1 \\
1
\end{array}\right)\left(\begin{array}{c}
1+1 \\
1
\end{array}\right) I & \ldots & 0 \\
\vdots & \vdots & \vdots & \vdots \\
I & (-1)^{1}\left(\begin{array}{c}
N \\
1
\end{array}\right)\left(\begin{array}{c}
N+1 \\
1
\end{array}\right) I & \ldots & (-1)^{N}\left(\begin{array}{c}
N \\
N
\end{array}\right)\left(\begin{array}{c}
N+N \\
N
\end{array}\right) I
\end{array}\right] \\
& \Gamma_{2 N}=\operatorname{diag}\left\{(-1)^{0} I,(-1)^{1} I, \cdots,(-1)^{N} I\right\} \Gamma_{1 N}, \\
& \Omega_{1 N}=\left[\begin{array}{cccccc}
I & -I & 0 & 0 & \cdots & 0 \\
0 & -I & I & 0 & \cdots & 0 \\
0 & -I & 0 & 2 I & \cdots & 0 \\
\vdots & \vdots & \vdots & \vdots & \vdots & \vdots \\
0 & -I & 0 & 0 & \cdots & N I
\end{array}\right], \Omega_{2 N}=\left[\begin{array}{cccccc}
I & -I & 0 & 0 & \cdots & 0 \\
I & 0 & -I & 0 & \cdots & 0 \\
I & 0 & 0 & -2 I & \cdots & 0 \\
\vdots & \vdots & \vdots & \vdots & \vdots & \vdots \\
I & 0 & 0 & 0 & \cdots & -N I
\end{array}\right] \\
& \phi_{1 N}(t)=\left[x^{T}(b), x^{T}(a), \rho_{1}^{T}(t), \rho_{2}^{T}(t), \cdots, \rho_{N}^{T}(t)\right]^{T}, \phi_{2 N}(t)=\left[x^{T}(b), x^{T}(a), \sigma_{1}^{T}(t), \sigma_{2}^{T}(t), \cdots, \sigma_{N}^{T}(t)\right]^{T} \\
& \text { with } \rho_{q}(t)=\frac{1}{(d-c)^{q}} \int_{c}^{d}(d-s)^{q-1} x(s) d s \text { and } \sigma_{q}(t)=\frac{1}{(d-c)^{q}} \int_{c}^{d}(s-c)^{q-1} x(s) d s,(q=1,2, \cdots, N) \text {. }
\end{aligned}
$$

\section{Main Results}

The stability and stabilization problem of system (9) are analyzed in this section by the memorybased coupling sampled-data switched control. To maintain the representation simplify the following notations are utilized:

$$
\begin{aligned}
\vartheta_{\alpha}(t)=\frac{1}{\left(t-t_{k}\right)^{\alpha}} \int_{t_{k}}^{t}(t-s)^{\alpha-1} x(s) d s, \gamma_{\alpha}(t)=\frac{1}{\left(t_{k+1}-t\right)^{\alpha}} \int_{t}^{t_{k+1}}(s-t)^{\alpha-1} x(s) d s \text { for } \alpha=1,2, \cdots, N . \\
\vartheta(t)=\left[\vartheta_{1}^{T}(t), \vartheta_{2}^{T}(t), \cdots, \vartheta_{N}^{T}(t)\right]^{T}, \gamma(t)=\left[\gamma_{1}^{T}(t), \gamma_{2}^{T}(t), \cdots, \gamma_{N}^{T}(t)\right]^{T}, \\
e_{s}=\left[0_{n \times(s-1) n}, I, 0_{n \times(2 N+7-s) n}\right], s=1,2, \cdots, 2 N+7, \\
\eta^{T}(t)=\left[x^{T}(t) x^{T}(t-\tau) \dot{x}^{T}(t) \dot{x}^{T}(t-\tau) x^{T}\left(t_{k}\right) x^{T}\left(t_{k+1}\right) x^{T}\left(t_{k}-\tau\right) \vartheta^{T}(t) \gamma^{T}(t)\right] .
\end{aligned}
$$

3.1. Stability Analysis. The following theorem is proposed to achieve the asymptotic stability of T-S fuzzy system (9). 
Theorem 1. For given gain matrices $K_{\mu, j}, L_{\mu, j}$, the scalars $\bar{d}>0, \tau, \beta$, and integer $N \geq 0$, system (9) is globally asymptotically stable for $d_{k} \in(0, \bar{d}]$, if there exists $P_{i}>0, S_{i}>0, U_{i}>0, X>0, Y>0$, symmetric matrices $Q_{i}, R_{i}$ and appropriate dimensional matrices $W_{i}, Z_{\iota}(\iota=1,2,3), J_{z N}(z=1,2)$ such that

$$
\begin{array}{r}
\dot{R}_{\delta} \leq 0, \\
\dot{P}_{\delta} \leq 0, \dot{Q}_{\delta} \leq 0, \dot{S}_{\delta} \leq 0, \dot{U}_{\delta} \leq 0, \dot{W}_{\delta} \leq 0, \\
{\left[\begin{array}{cc}
\Xi_{\left(d_{k}, 0\right)} & \sqrt{d_{k}} \Lambda_{2 N}^{T} J_{2 N}^{T} \\
* & -Y_{N}
\end{array}\right]<0,} \\
{\left[\begin{array}{cc}
\Xi_{\left(d_{k}, d_{k}\right)} & \sqrt{d_{k}} \Lambda_{1 N}^{T} J_{1 N}^{T} \\
* & -X_{N}
\end{array}\right]<0,}
\end{array}
$$

hold, where

$$
\begin{aligned}
\Xi_{\left(d_{k}, d(t)\right)=} & \operatorname{ym}\left\{e_{1}^{T} P_{i} e_{3}\right\}-\left(e_{1}-e_{5}\right)^{T} Q_{i}\left(e_{1}-e_{5}\right)+\operatorname{Sym}\left\{\left(d_{k}-d(t)\right)\left(e_{1}-e_{5}\right)^{T} Q_{i} e_{3}\right\} \\
& +\tau e_{1}^{T} S_{i} e_{1}-\tau e_{2}^{T} S_{i} e_{2}-d(t)\left[\begin{array}{l}
e_{5} \\
e_{6} \\
e_{7}
\end{array}\right] R_{i}^{T}\left[\begin{array}{l}
e_{5} \\
e_{6} \\
e_{7}
\end{array}\right]+\operatorname{Sym}\left\{d(t) e_{3}^{T} W_{i}\left[\begin{array}{l}
e_{5} \\
e_{6}
\end{array}\right]\right\} \\
& +\left(d_{k}-d(t)\right)\left[\begin{array}{l}
e_{5} \\
e_{6} \\
e_{7}
\end{array}\right]^{T} R_{i}\left[\begin{array}{l}
e_{5} \\
e_{6} \\
e_{7}
\end{array}\right]-e_{4}^{T} U_{i} e_{4}+e_{3}^{T} U_{i} e_{3}+\operatorname{Sym}\left\{\Lambda_{1 N}^{T} J_{1 N}^{T} \Pi_{1 N} \Lambda_{1 N}\right\} \\
& +\operatorname{Sym}\left\{\Lambda_{2 N}^{T} J_{2 N}^{T} \Pi_{2 N} \Lambda_{2 N}\right\}+\operatorname{Sym}\left\{\left(e_{1}-e_{6}\right)^{T} W_{i}\left[e_{5}\right]\right\}+\left(d_{k}-d(t)\right) e_{3}^{T} X e_{3} \\
& +d(t) e_{3}^{T} Y e_{3}+\operatorname{Sym}\left\{\Upsilon\left(-e_{3}+A_{i} e_{1}+\beta B_{i} K_{\mu, j} e_{7}+(1-\beta) B_{i} L_{\mu, j} e_{5}\right)\right\}, \\
\Lambda_{1 N}= & {\left[e_{1}^{T}, e_{5}^{T}, e_{8}^{T}, e_{9}^{T}, \cdots, e_{N+7}^{T}\right]^{T}, \Lambda_{2 N}=\left[e_{6}^{T}, e_{1}^{T}, e_{N+8}^{T}, e_{N+9}^{T}, \cdots, e_{2 N+7}^{T}\right]^{T}, } \\
X_{N}= & \operatorname{diag}\{X, 3 X, \cdots,(2 N+1) X\}, Y_{N}=\operatorname{diag}\{Y, 3 Y, \cdots,(2 N+1) Y\}, \\
\Upsilon= & e_{1}^{T} Z_{1}^{T}+e_{3}^{T} Z_{2}^{T}+e_{7}^{T} Z_{3}^{T} .
\end{aligned}
$$


Proof Let us choose the following LKF:

$$
V(t)=\sum_{l=1}^{6} V_{l}(t), \quad t \in\left[t_{k}, t_{k+1}\right)
$$

where

$$
\begin{aligned}
& V_{1}(t)=\sum_{i=1}^{r} \delta_{i}(w(t)) x^{T}(t) P_{i} x(t) \\
& V_{2}(t)=\left(d_{k}-d(t)\right) \sum_{i=1}^{r} \delta_{i}(w(t))\left[x(t)-x\left(t_{k}\right)\right]^{T} Q_{i}\left[x(t)-x\left(t_{k}\right)\right] \\
& V_{3}(t)=2 d(t) \sum_{i=1}^{r} \delta_{i}(w(t))\left[x(t)-x\left(t_{k+1}\right)\right]^{T} W_{i}\left[\begin{array}{c}
x\left(t_{k}\right) \\
x\left(t_{k+1}\right)
\end{array}\right] \\
& V_{4}(t)=\left(d_{k}-d(t)\right) d(t) \sum_{i=1}^{r} \delta_{i}(w(t))\left[\begin{array}{c}
x\left(t_{k}\right) \\
x\left(t_{k+1}\right) \\
x\left(t_{k}-\tau\right)
\end{array}\right]^{T} R_{i}\left[\begin{array}{c}
x\left(t_{k}\right) \\
x\left(t_{k+1}\right) \\
x\left(t_{k}-\tau\right)
\end{array}\right] \\
& V_{5}(t)=\tau \sum_{i=1}^{r} \delta_{i}(w(t)) \int_{t-\tau}^{t} x^{T}(s) S_{i} x(s) d s+\sum_{i=1}^{r} \delta_{i}(w(t)) \int_{t-\tau}^{t} \dot{x}^{T}(s) U_{i} \dot{x}(s) d s \\
& V_{6}(t)=\left(d_{k}-d(t)\right) \int_{t_{k}}^{t} \dot{x}^{T}(s) X \dot{x}(s) d s-d(t) \int_{t}^{t_{k+1}} \dot{x}^{T}(s) Y \dot{x}(s) d s .
\end{aligned}
$$

The time derivative of 15 is written as

$$
\begin{aligned}
\dot{V}_{1}(t)= & 2 x^{T}(t) P_{\delta} \dot{x}(t)+x^{T}(t) \dot{P}_{\delta} x(t) \\
\dot{V}_{2}(t)= & -\left(x(t)-x\left(t_{k}\right)\right)^{T} Q_{\delta}\left(x(t)-x\left(t_{k}\right)\right)+2\left(d_{k}-d(t)\right)\left(x(t)-x\left(t_{k}\right)\right)^{T} Q_{\delta} \dot{x}(t) \\
& +\left(d_{k}-d(t)\right)\left(x(t)-x\left(t_{k}\right)\right)^{T} \dot{Q}_{\delta}\left(x(t)-x\left(t_{k}\right)\right) \\
\dot{V}_{3}(t)= & 2\left[x(t)-x\left(t_{k+1}\right)\right]^{T} W_{\delta}\left[\begin{array}{c}
x\left(t_{k}\right) \\
x\left(t_{k+1}\right)
\end{array}\right]+2 d(t) \dot{x}^{T}(t) W_{\delta}\left[\begin{array}{c}
x\left(t_{k}\right) \\
x\left(t_{k+1}\right)
\end{array}\right] \\
& +2 d(t)\left[x(t)-x\left(t_{k+1}\right)\right]^{T} \dot{W}_{\delta}\left[\begin{array}{c}
x\left(t_{k}\right) \\
x\left(t_{k+1}\right)
\end{array}\right]
\end{aligned}
$$


Fuzzy membership function dependent switched control for nonlinear systems with memory sampled-data information 11

$$
\begin{aligned}
& \dot{V}_{4}(t)=-d(t)\left[\begin{array}{c}
x\left(t_{k}\right) \\
x\left(t_{k+1}\right) \\
x\left(t_{k}-\tau\right)
\end{array}\right]^{T} R_{\delta}\left[\begin{array}{c}
x\left(t_{k}\right) \\
x\left(t_{k+1}\right) \\
x\left(t_{k}-\tau\right)
\end{array}\right]+\left(d_{k}-d(t)\right)\left[\begin{array}{c}
x\left(t_{k}\right) \\
x\left(t_{k+1}\right) \\
x\left(t_{k}-\tau\right)
\end{array}\right]^{T} R_{\delta}\left[\begin{array}{c}
x\left(t_{k}\right) \\
x\left(t_{k+1}\right) \\
x\left(t_{k}-\tau\right)
\end{array}\right] \\
& +\left(d_{k}-d(t)\right) d(t)\left[\begin{array}{c}
x\left(t_{k}\right) \\
x\left(t_{k+1}\right) \\
x\left(t_{k}-\tau\right)
\end{array}\right]^{T}\left[\begin{array}{c}
x\left(t_{k}\right) \\
x\left(t_{k+1}\right) \\
x\left(t_{k}-\tau\right)
\end{array}\right] \\
& \dot{V}_{5}(t)=\tau\left[x^{T}(t) S_{\delta} x(t)-x^{T}(t-\tau) S_{\delta} x(t-\tau)\right]+\dot{x}^{T}(t) U_{\delta} \dot{x}(t)-\dot{x}^{T}(t-\tau) U_{\delta} \dot{x}(t-\tau) \\
& +\tau \int_{t-\tau}^{t} x^{T}(s) \dot{S}_{\delta} x(s) d s+\int_{t-\tau}^{t} \dot{x}^{T}(s) \dot{U}_{\delta} \dot{x}(s) d s \\
& \dot{V}_{6}(t)=\left(d_{k}-d(t)\right) \dot{x}^{T}(t) X \dot{x}(t)+d(t) \dot{x}^{T}(t) Y \dot{x}(t)-\int_{t_{k}}^{t} \dot{x}^{T}(s) X \dot{x}(s) d s-\int_{t}^{t_{k+1}} \dot{x}^{T}(s) Y \dot{x}(s) d s .
\end{aligned}
$$

Applying the Lemma 2 for the integral terms of (21), we get

$$
\begin{aligned}
& -\int_{t_{k}}^{t} \dot{x}^{T}(s) X \dot{x}(s) d s \leq \eta^{T}(t)\left[\operatorname{Sym}\left\{\Lambda_{1 N}^{T} J_{1 N}^{T} \Pi_{1 N} \Lambda_{1 N}\right\}+d(t) \Lambda_{1 N}^{T} J_{1 N}^{T} X_{N}^{-1} J_{1 N} \Lambda_{1 N}\right] \eta(t) \\
& -\int_{t}^{t_{k+1}} \dot{x}^{T}(s) Y \dot{x}(s) d s \leq \eta^{T}(t)\left[\operatorname{Sym}\left\{\Lambda_{2 N}^{T} J_{2 N}^{T} \Pi_{2 N} \Lambda_{2 N}\right\}+\left(d_{k}-d(t)\right) \Lambda_{2 N}^{T} J_{2 N}^{T} Y_{N}^{-1} J_{2 N} \Lambda_{2 N}\right] \eta(t) .
\end{aligned}
$$

Now, for any appropriate dimensional matrices $Z_{1}, Z_{2}, Z_{3}$, we have the following equation:

$2 \sum_{i=1}^{r} \sum_{j=1}^{r} \delta_{i}(w(t)) \delta_{j}\left(w\left(t_{k}\right)\right) \xi(t)\left[-\dot{x}(t)+A_{i} x(t)+\beta(t) B_{i} K_{\mu, j} x\left(t_{k}-\tau\right)+(1-\beta(t)) B_{i} L_{\mu, j} x\left(t_{k}\right)\right]=0$,

with $\xi(t)=x^{T}(t) Z_{1}^{T}+\dot{x}^{T}(t) Z_{2}^{T}+x^{T}\left(t_{k}-\tau\right) Z_{3}^{T}$.

Combining (16)-24 and taking expectations, we get

$$
\begin{aligned}
\mathbb{E}\{\dot{V}(t)\} & \leq \sum_{i=1}^{r} \sum_{j=1}^{r} \delta_{i}(w(t)) \delta_{j}\left(w\left(t_{k}\right)\right) \eta^{T}(t) \bar{\Xi}_{\left(d_{k}, d(t)\right)} \eta(t) \\
& =\sum_{i=1}^{r} \sum_{j=1}^{r} \delta_{i}(w(t)) \delta_{j}\left(w\left(t_{k}\right)\right) \eta^{T}(t)\left[\frac{d_{k}-d(t)}{d_{k}} \bar{\Xi}_{\left(d_{k}, 0\right)}+\frac{d(t)}{d_{k}} \bar{\Xi}_{\left(d_{k}, d_{k}\right)}\right] \eta(t),
\end{aligned}
$$


where $\bar{\Xi}_{\left(d_{k}, d(t)\right)}=\Xi_{\left(d_{k}, d(t)\right)}+\left(d_{k}-d(t)\right) \Lambda_{2 N}^{T} J_{2 N}^{T} Y_{N}^{-1} J_{2 N} \Lambda_{2 N}+d(t) \Lambda_{1 N}^{T} J_{1 N}^{T} X_{N}^{-1} J_{1 N} \Lambda_{1 N}$, here $\bar{\Xi}_{\left(d_{k}, d(t)\right)}$ is a convex combination of $\bar{\Xi}_{\left(d_{k}, 0\right)}$ and $\bar{\Xi}_{\left(d_{k}, d_{k}\right)}$. Then $\bar{\Xi}_{\left(d_{k}, d(t)\right)}<0$ if and only if 13)-14 hold.

3.2. Controller Design. In this subsection, we will analyze the stabilization of system (9) via memory-based coupling SDC by the results in Theorem 1.

Theorem 2. For given scalars $\tau, \beta, \bar{d}>0, \lambda_{k}(k=1,2)$ and nonnegative integer $N$, the system (9) is globally asymptotically stable under the memory-based coupling sampled-data switched control (8) for $d_{k} \in(0, \bar{d}]$, if there exists $\widetilde{P}_{i}>0, \widetilde{S}_{i}>0, \widetilde{U}_{i}>0, \widetilde{X}>0, \widetilde{Y}>0$, symmetric matrices $\widetilde{Q}_{i}$, $\widetilde{R}_{i}$, any nonsingular matrix $Z$ and any appropriate dimensional matrices $\widetilde{W}_{i}, \widetilde{J}_{z N}(z=1,2), G_{\mu, j}, H_{\mu, j}$ such that the following inequalities are satisfied

$$
\begin{array}{r}
\dot{\widetilde{P}}_{\delta} \leq 0, \dot{\widetilde{Q}}_{\delta} \leq 0, \dot{\widetilde{S}}_{\delta} \leq 0, \dot{\widetilde{U}}_{\delta} \leq 0, \dot{\widetilde{W}}_{\delta} \leq 0, \\
{\left[\begin{array}{cc}
\widetilde{\Xi}_{\left(d_{k}, 0\right)} & \sqrt{d_{k}} \Lambda_{2 N}^{T} \widetilde{J}_{2 N}^{T} \\
* & -\widetilde{Y}_{N}
\end{array}\right]<0,} \\
{\left[\begin{array}{cc}
\widetilde{\Xi}_{\left(d_{k}, d_{k}\right)} & \sqrt{d_{k}} \Lambda_{1 N}^{T} \widetilde{J}_{1 N}^{T} \\
* & -\widetilde{X}_{N}
\end{array}\right]<0,} \\
\end{array}
$$

where

$$
\begin{aligned}
\widetilde{\Xi}_{\left(d_{k}, d(t)\right)}= & \operatorname{Sym}\left\{e_{1}^{T} \widetilde{P}_{i} e_{3}\right\}-\left(e_{1}-e_{5}\right)^{T} \widetilde{Q}_{i}\left(e_{1}-e_{5}\right)+\operatorname{Sym}\left\{\left(d_{k}-d(t)\right)\left(e_{1}-e_{5}\right)^{T} \widetilde{Q}_{i} e_{3}\right\} \\
& +\tau e_{1}^{T} \widetilde{S}_{i} e_{1}-\tau e_{2}^{T} \widetilde{S}_{i} e_{2}+d(t) e_{3}^{T} \widetilde{Y} e_{3}-d(t)\left[\begin{array}{l}
e_{5} \\
e_{6} \\
e_{7}
\end{array}\right] \widetilde{R}_{i}^{T}\left[\begin{array}{l}
e_{5} \\
e_{6} \\
e_{7}
\end{array}\right]+\operatorname{Sym}\left\{d(t) e_{3}^{T} \widetilde{W}_{i}\left[\begin{array}{l}
e_{5} \\
e_{6}
\end{array}\right]\right\} \\
& +\left(d_{k}-d(t)\right)\left[\begin{array}{l}
e_{5} \\
e_{6} \\
e_{7}
\end{array}\right]^{T} \widetilde{R}_{i}\left[\begin{array}{c}
e_{5} \\
e_{6} \\
e_{7}
\end{array}\right]-e_{4}^{T} \widetilde{U}_{i} e_{4}+e_{3}^{T} \widetilde{U}_{i} e_{3}+\operatorname{Sym}\left\{\Lambda_{1 N}^{T} \widetilde{J}_{1 N}^{T} \Pi_{1 N} \Lambda_{1 N}\right\} \\
& +\left(d_{k}-d(t)\right) e_{3}^{T} \widetilde{X} e_{3}+\operatorname{Sym}\left\{\Lambda_{2 N}^{T} \widetilde{J}_{2 N}^{T} \Pi_{2 N} \Lambda_{2 N}\right\}+\operatorname{Sym}\left\{\left(e_{1}-e_{6}\right)^{T} \widetilde{W}_{i}\left[\begin{array}{l}
e_{5} \\
e_{6}
\end{array}\right]\right\}
\end{aligned}
$$


Fuzzy membership function dependent switched control for nonlinear systems with memory sampled-data information 13

$$
\begin{aligned}
& +\operatorname{Sym}\left\{\widetilde{\Upsilon}\left(-Z e_{3}+A_{i} Z e_{1}+\beta B_{i} G_{\mu, j} e_{7}+(1-\beta) B_{i} H_{\mu, j} e_{5}\right)\right\}, \\
\Lambda_{1 N}= & {\left[e_{1}^{T}, e_{5}^{T}, e_{8}^{T}, e_{9}^{T}, \cdots, e_{N+7}^{T}\right]^{T}, \Lambda_{2 N}=\left[e_{6}^{T}, e_{1}^{T}, e_{N+8}^{T}, e_{N+9}^{T}, \cdots, e_{2 N+7}^{T}\right]^{T}, } \\
\widetilde{X}_{N}= & \operatorname{diag}\{\widetilde{X}, 3 \widetilde{X}, \cdots,(2 N+1) \widetilde{X}\}, \widetilde{Y}_{N}=\operatorname{diag}\{\widetilde{Y}, 3 \widetilde{Y}, \cdots,(2 N+1) \widetilde{Y}\}, \\
\widetilde{\Upsilon}= & e_{1}^{T}+\lambda_{1} e_{3}^{T}+\lambda_{2} e_{7}^{T} .
\end{aligned}
$$

Moreover, the gain matrices are obtained by $K_{\mu, j}=G_{\mu, j} Z^{-1}$, and $L_{\mu, j}=H_{\mu, j} Z^{-1}$.

Proof Define $Z_{1}=Z^{-1}, Z_{2}=\lambda_{1} Z^{-1}, Z_{3}=\lambda_{2} Z^{-1}, \widetilde{P}_{i}=Z^{T} P_{i} Z, G_{\mu, j}=K_{\mu, j} Z, H_{\mu, j}=L_{\mu, j} Z$, $\widetilde{S}_{i}=Z^{T} S_{i} Z, \widetilde{U}_{i}=Z^{T} U_{i} Z, \tilde{X}=Z^{T} X Z, \widetilde{Y}=Z^{T} Y Z, \widetilde{Q}_{i}=Z^{T} Q_{i} Z, \widetilde{W}_{i}=Z^{T} W_{i} \operatorname{diag}\{Z, Z\}$, $\widetilde{J}_{1 N}=Z^{T} J_{1 N} Z, \widetilde{J}_{2 N}=Z^{T} J_{2 N} Z, \widetilde{R}_{i}=\operatorname{diag}\left\{Z^{T}, Z^{T}, Z^{T}\right\} R_{i} \operatorname{diag}\{Z, Z, Z\}$. Pre and post multiply 11 by $\operatorname{diag}\left\{Z^{T}, Z^{T}, Z^{T}\right\}$ and its transpose, we get 26). The inequality 12 is pre and post multiplied by $Z^{T}$ and $Z$ respectively, we have (27). The inequalities 13 and 14 are pre and post multiplied by $\operatorname{diag}\{\underbrace{Z^{T}, Z^{T}, \cdots, Z^{T}}_{2 N+7 \text { times }}\}$ and its transpose respectively, we obtain (28) and (29).

Remark 2. In the existing studies, the novel integral inequalities have been utilized to obtain the less conservatism for stabilization of T-S fuzzy systems, such as Jensen's inequality [17], Wirtinger's inequality [14, 23], second-order Bessel inequality [30]. Different from those studies, the generalized $N$-order free-matrix-based inequality is handle the stabilization problem of T-S fuzzy systems in the present study, which has some inequalities as particular cases. For example, substituting $N=0$ in 10, it can be reduced to Jensen's inequality which has been utilized for T-S fuzzy systems in [17]. Similarly, when $N=1$, the inequality becomes Wirtinger's inequality as in [14, 23]. Moreover, the second-order Bessel inequality $(N=2)$ has been examined in [30]. Thus, the proposed method utilizes the generalized $N$-order free-matrix-based inequality which is more general than the aforesaid previous studies.

Remark 3. It should be pointed out that the purpose of the following corollary is to affirm the advantage of the proposed method under conventional SDC input, that is, the stochastic variable $\beta(t)$ vanishes in (4)

$$
u(t)=\sum_{j=1}^{r} \delta_{j}\left(w\left(t_{k}\right)\right) L_{j} x\left(t_{k}\right) .
$$


Hence, the corresponding closed-loop system (9) is presented as follows:

$$
\dot{x}(t)=\sum_{i=1}^{r} \sum_{j=1}^{r} \delta_{i}(w(t)) \delta_{j}\left(w\left(t_{k}\right)\right)\left[A_{i} x(t)+B_{i} L_{\mu, j} x\left(t_{k}\right)\right], \quad t_{k} \leq t<t_{k+1} .
$$

Also, the LKF can be constructed from (15) without the constant signal transmission delay and the stochastic variable as follows:

$$
V(t)=\sum_{l=1}^{5} V_{l}(t), \quad t \in\left[t_{k}, t_{k+1}\right)
$$

where

$$
\begin{aligned}
& V_{1}(t)=\sum_{i=1}^{r} \delta_{i}(w(t)) x^{T}(t) P_{i} x(t) \\
& V_{2}(t)=\left(d_{k}-d(t)\right) \sum_{i=1}^{r} \delta_{i}(w(t))\left[x(t)-x\left(t_{k}\right)\right]^{T} Q_{i}\left[x(t)-x\left(t_{k}\right)\right] \\
& V_{3}(t)=2 d(t) \sum_{i=1}^{r} \delta_{i}(w(t))\left[x(t)-x\left(t_{k+1}\right)\right]^{T} W_{i}\left[\begin{array}{c}
x\left(t_{k}\right) \\
x\left(t_{k+1}\right)
\end{array}\right] \\
& \left.V_{4}(t)=\left(d_{k}-d(t)\right) d(t) \sum_{i=1}^{r} \delta_{i}(w(t))\left[x\left(t_{k}\right)\right]^{T} t_{k+1}\right) R^{t_{i}}\left[x\left(t_{k}\right)\right. \\
& \left.\left.V_{5}(t)=\left(d_{k}-d(t)\right) \int_{t_{k}}^{t} \dot{x}^{T}(s) X \dot{x}(s) d s-d(t) \int_{t}^{t_{k+1}}\right)\right]
\end{aligned}
$$

3.3. Stabilization of T-S Fuzzy Systems under Conventional SDC:. In this subsection, we derive the adequate conditions to assure the stabilization of T-S fuzzy systems under conventional SDC scheme which is a special case of previous subsection.

Corollary 1. For given scalars $\lambda, \bar{d}>0$, and nonnegative integer $N$, system 31 is globally asymptotically stable by SDC (30), if there exists $\widetilde{P}_{i}>0, \widetilde{X}>0, \widetilde{Y}>0, \widetilde{Q}_{i}=\widetilde{Q}_{i}^{T}, \widetilde{R}_{i}=\widetilde{R}_{i}^{T}$, nonsingular matrix $Z$, and appropriate dimensional matrices $\widetilde{W}_{i}, \widetilde{J}_{z N}(z=1,2), H_{\mu, j}$ such that

$$
\begin{array}{r}
\dot{\widetilde{P}}_{\delta} \leq 0, \dot{\widetilde{Q}}_{\delta} \leq 0, \dot{\widetilde{R}}_{\delta} \leq 0, \dot{\widetilde{W}}_{\delta} \leq 0, \\
{\left[\begin{array}{cc}
\widetilde{\Xi}_{\left(d_{k}, 0\right)} & \sqrt{d_{k}} \Lambda_{2 N}^{T} \widetilde{J}_{2 N}^{T} \\
* & -\widetilde{Y}_{N}
\end{array}\right]<0,}
\end{array}
$$


Fuzzy membership function dependent switched control for nonlinear systems with memory sampled-data information 15

$$
\left[\begin{array}{cc}
\widetilde{\Xi}_{\left(d_{k}, d_{k}\right)} & \sqrt{d_{k}} \Lambda_{1 N}^{T} \widetilde{J}_{1 N}^{T} \\
* & -\widetilde{X}_{N}
\end{array}\right]<0,
$$

hold for $d_{k} \in(0, \bar{d}]$. Here,

$$
\begin{aligned}
& \widetilde{\Xi}_{\left(d_{k}, d(t)\right)}=\operatorname{Sym}\left\{e_{1}^{T} \widetilde{P}_{i} e_{2}\right\}-\left(e_{1}-e_{3}\right)^{T} \widetilde{Q}_{i}\left(e_{1}-e_{3}\right)+\operatorname{Sym}\left\{\left(d_{k}-d(t)\right)\left(e_{1}-e_{3}\right)^{T} \widetilde{Q}_{i} e_{2}\right\} \\
& +d(t) e_{3}^{T} \widetilde{Y} e_{3}-d(t)\left[\begin{array}{l}
e_{3} \\
e_{4}
\end{array}\right]^{T} \widetilde{R}_{i}\left[\begin{array}{l}
e_{3} \\
e_{4}
\end{array}\right]+\left(d_{k}-d(t)\right)\left[\begin{array}{l}
e_{3} \\
e_{4}
\end{array}\right]^{T} \widetilde{R}_{i}\left[\begin{array}{l}
e_{3} \\
e_{4}
\end{array}\right] \\
& +\left(d_{k}-d(t)\right) e_{3}^{T} \widetilde{X} e_{3}+\operatorname{Sym}\left\{\Lambda_{1 N}^{T} \widetilde{J}_{1 N}^{T} \Pi_{1 N} \Lambda_{1 N}\right\}+\operatorname{Sym}\left\{\Lambda_{2 N}^{T} \widetilde{J}_{2 N}^{T} \Pi_{2 N} \Lambda_{2 N}\right\} \\
& +\operatorname{Sym}\left\{d(t) e_{2}^{T} \widetilde{W}_{i}\left[\begin{array}{l}
e_{3} \\
e_{4}
\end{array}\right]\right\}+\operatorname{Sym}\left\{\left(e_{1}-e_{4}\right)^{T} \widetilde{W}_{i}\left[\begin{array}{l}
e_{3} \\
e_{4}
\end{array}\right]\right\} \\
& +\operatorname{Sym}\left\{\widetilde{\Upsilon}\left(-Z e_{2}+A_{i} Z e_{1}+B_{i} H_{\mu, j} e_{3}\right)\right\}, \\
& \Lambda_{1 N}=\left[e_{1}^{T}, e_{3}^{T}, e_{5}^{T}, e_{6}^{T}, \cdots, e_{N+4}^{T}\right]^{T}, \Lambda_{2 N}=\left[e_{4}^{T}, e_{1}^{T}, e_{N+5}^{T}, e_{N+6}^{T}, \cdots, e_{2 N+4}^{T}\right]^{T}, \\
& \widetilde{\Upsilon}=e_{1}^{T}+\lambda e_{2}^{T}, e_{s}=\left[0_{n \times(s-1) n}, I, 0_{n \times(2 N+4-s) n}\right], s=1,2, \cdots, 2 N+4 \\
& \widetilde{X}_{N}=\operatorname{diag}\{\widetilde{X}, 3 \widetilde{X}, \cdots,(2 N+1) \widetilde{X}\}, \widetilde{Y}_{N}=\operatorname{diag}\{\widetilde{Y}, 3 \widetilde{Y}, \cdots,(2 N+1) \widetilde{Y}\} .
\end{aligned}
$$

The control gain matrices are calculated by $L_{\mu, j}=H_{\mu, j} Z^{-1}$.

Proof With a similar procedure as Theorem (1) and (2), the proof can be derived.

\section{Numerical ExAMPLES}

This section presents the superiority of derived theoretical results by using two numerical examples.

Example 1. The dynamics of Lorenz system described as follows [14]:

$$
\left\{\begin{array}{l}
\dot{x}_{1}(t)=a\left(x_{2}(t)-x_{1}(t)\right) \\
\dot{x}_{2}(t)=c x_{1}(t)-x_{2}(t)-x_{1}(t) x_{3}(t)+u_{1}(t) \\
\dot{x}_{3}(t)=x_{1}(t) x_{2}(t)-b x_{3}(t)+u_{2}(t)
\end{array}\right.
$$

where $x_{1}(t), x_{2}(t), x_{3}(t)$ are the state variables with $x_{1}(t) \in[-d, d]$ and $a=10, d=25 b=8 / 3$, and $c=28$, are known constants. The Lorenz system (36) can be reformulated as T-S fuzzy model (9) 
with

$$
A_{1}=\left[\begin{array}{ccc}
-a & a & 0 \\
c & -1 & -d \\
0 & d & -b
\end{array}\right], A_{2}=\left[\begin{array}{ccc}
-a & a & 0 \\
c & -1 & d \\
0 & -d & -b
\end{array}\right], B_{1}=B_{2}=\left[\begin{array}{ll}
0 & 0 \\
1 & 0 \\
0 & 1
\end{array}\right],
$$

The membership functions are chosen as $\delta_{1}\left(x_{1}(t)\right)=(1 / 2)\left(1+\left(x_{1}(t) / d\right)\right)$ and $\delta_{2}\left(x_{1}(t)\right)=1-\delta_{1}\left(x_{1}(t)\right)$. Then, the trajectories of Lorenz system without control input is plotted in Figure 2 and we conclude that it is unstable. So, we have to find the effective controller to stabilize the system (36). Choosing

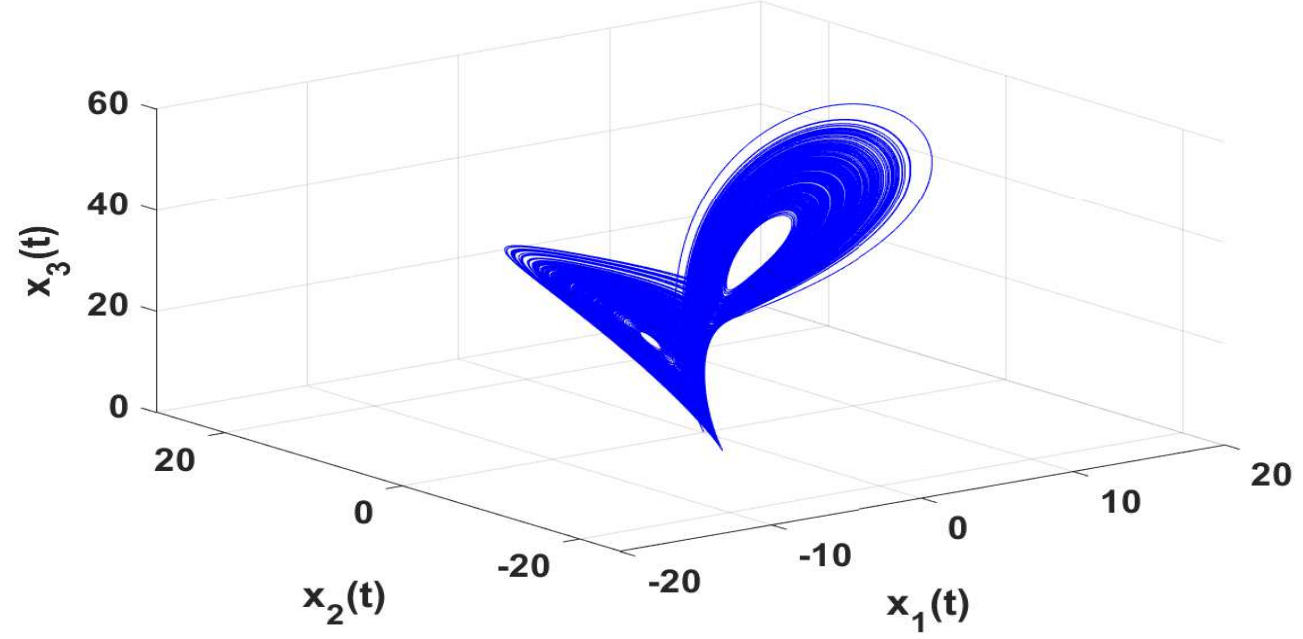

Figure 2. State trajectories of system (36) without control input.

$\tau=0.02, \beta=0.5, \lambda_{1}=\lambda_{2}=1$ and from the switching rule (6), solving the LMIs $260-(29)$ for the constraints $\mathbb{B}_{1}:\left\{\widetilde{P}_{1}>\widetilde{P}_{2}, \widetilde{S}_{1}>\widetilde{S}_{2}, \widetilde{Q}_{1}>\widetilde{Q}_{2}, \widetilde{R}_{1}>\widetilde{R}_{2}, \widetilde{U}_{1}>\widetilde{U}_{2}, \widetilde{W}_{1}>\widetilde{W}_{2}\right\}$, we obtain the control gain matrices with the LUB of sampling interval $\bar{d}=0.0152$ as follows:

$$
\begin{aligned}
& K_{1,1}=\left[\begin{array}{ccc}
1.1050 & -25.2813 & -31.1077 \\
-0.7818 & 26.0039 & -8.7913
\end{array}\right], K_{1,2}=\left[\begin{array}{ccc}
0.7630 & -20.9267 & -27.3992 \\
-0.6785 & 22.2514 & -7.8551
\end{array}\right], \\
& L_{1,1}=\left[\begin{array}{ccc}
-50.5547 & -141.0197 & -154.8959 \\
-4.4431 & 141.6054 & -42.4118
\end{array}\right], L_{1,2}=\left[\begin{array}{ccc}
-50.1370 & -134.4566 & -144.7487 \\
-3.9263 & 129.4616 & -41.5925
\end{array}\right] \text {. }
\end{aligned}
$$

Similarly, for the same parameter values, we obtain the LUB of sampling interval $\bar{d}=0.0102$ under the constraints $\mathbb{B}_{2}:\left\{\widetilde{P}_{1} \leq \widetilde{P}_{2}, \widetilde{S}_{1} \leq \widetilde{S}_{2}, \widetilde{Q}_{1} \leq \widetilde{Q}_{2}, \widetilde{R}_{1} \leq \widetilde{R}_{2}, \widetilde{U}_{1} \leq \widetilde{U}_{2}, \widetilde{W}_{1} \leq \widetilde{W}_{2}\right\}$. In addition, the 
Fuzzy membership function dependent switched control for nonlinear systems with memory sampled-data information 17

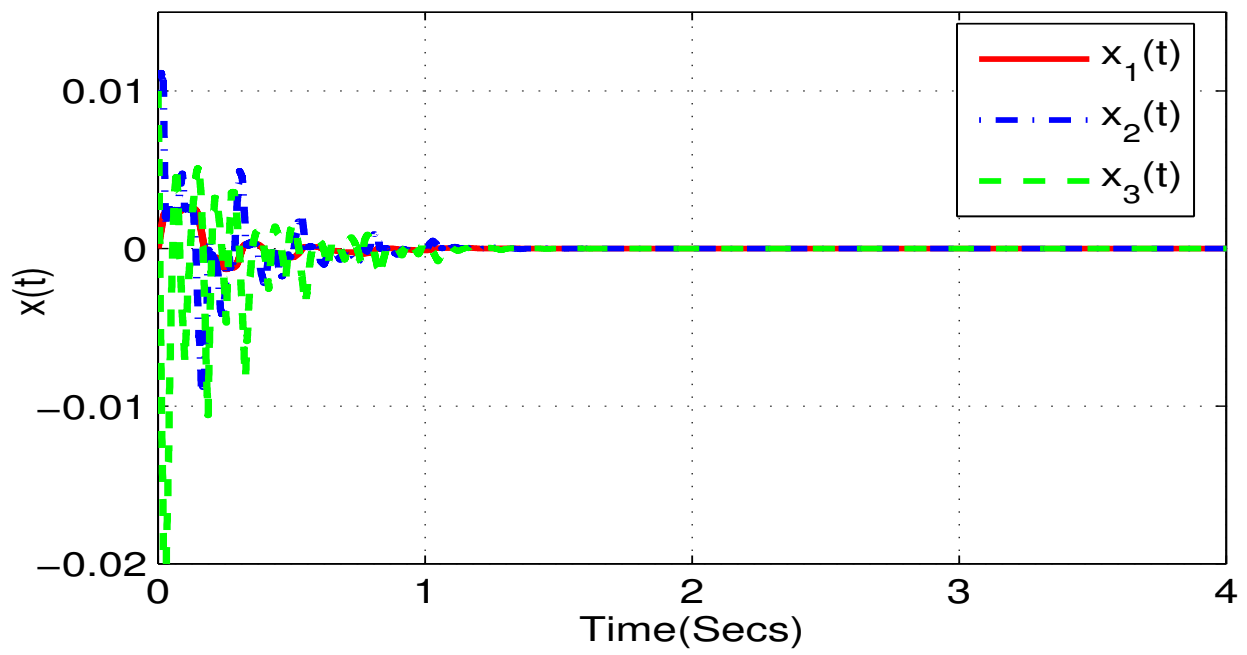

Figure 3. State responses of system (36).

control gains are calculated as follows

$$
\begin{aligned}
& K_{2,1}=\left[\begin{array}{ccc}
0.6197 & -16.2857 & 19.8346 \\
0.8852 & -21.5683 & -15.2000
\end{array}\right], K_{2,2}=\left[\begin{array}{ccc}
0.7282 & -17.2508 & 18.5655 \\
1.0978 & -27.6317 & -16.9513
\end{array}\right], \\
& L_{2,1}=\left[\begin{array}{ccc}
-50.6868 & -85.8608 & 115.4872 \\
7.4194 & -162.1230 & -119.0891
\end{array}\right], L_{2,2}=\left[\begin{array}{ccc}
-50.1046 & -96.6959 & 110.1537 \\
7.4990 & -175.8157 & -119.1089
\end{array}\right] \text {. }
\end{aligned}
$$

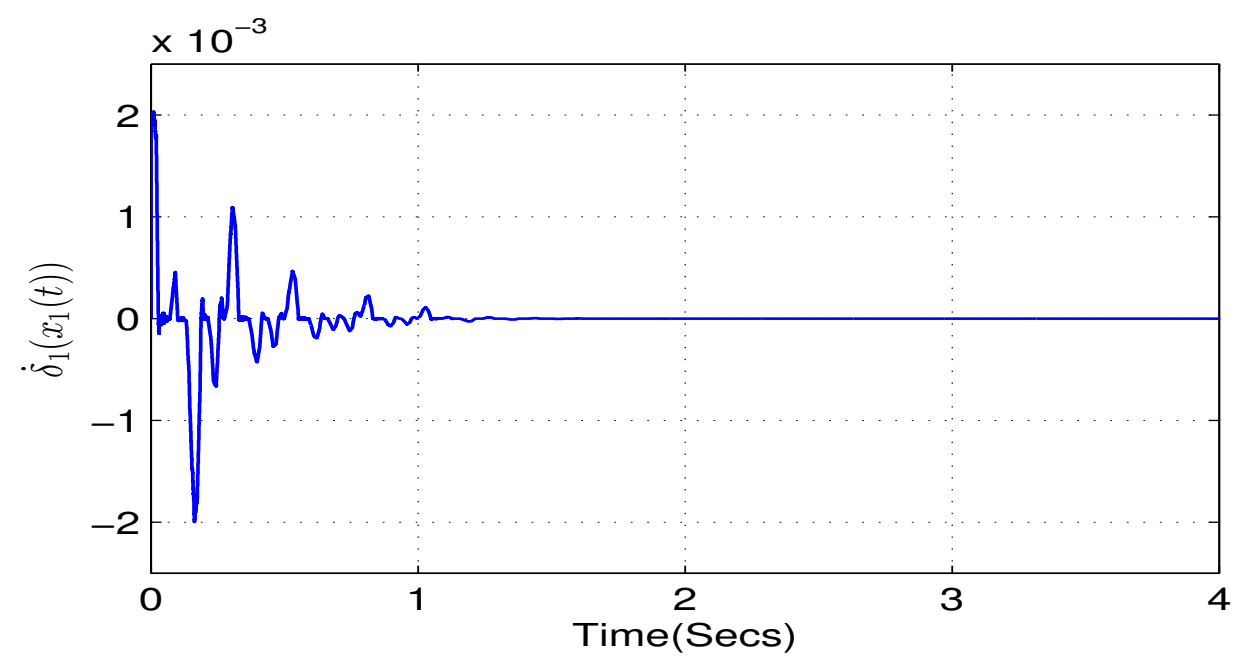

FIGURE 4. The evolution of $\dot{\delta}_{1}\left(x_{1}(t)\right)$. 


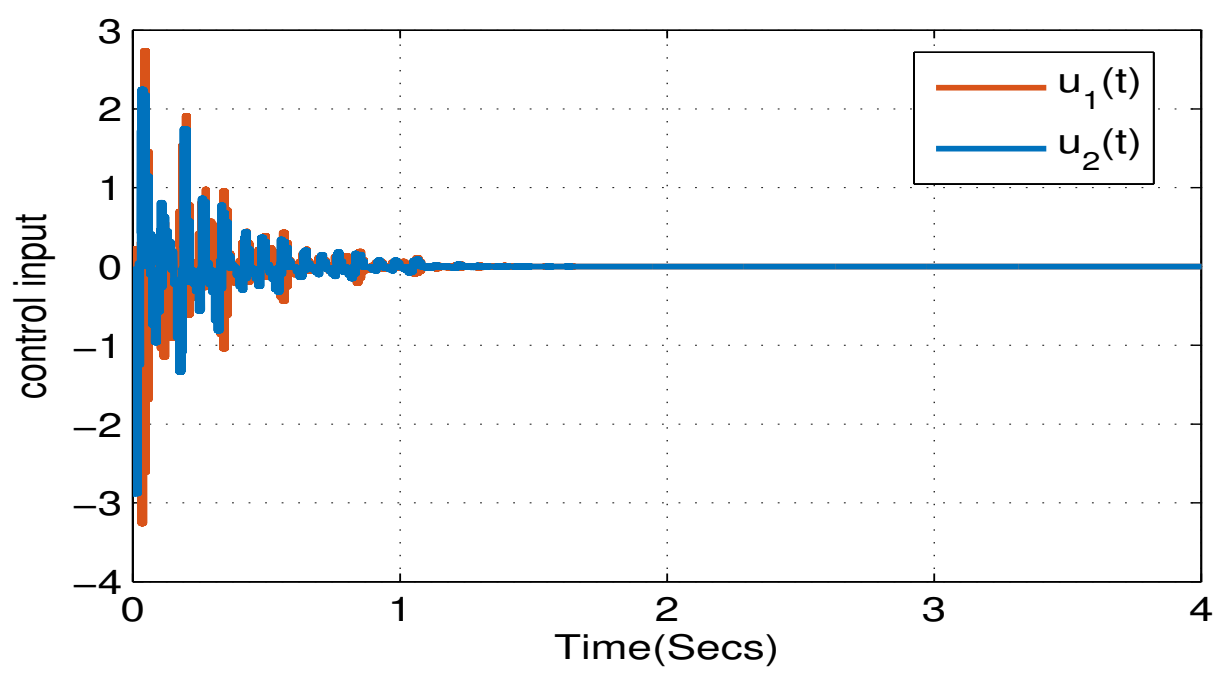

Figure 5. The trajectory of $u(t)$ in Example 1.

Hence, the final LUB of sampling interval is obtained as $\bar{d}=0.0102$. Moreover, the state trajectories of the closed-loop system are displayed in Figure 3, which is asymptotically stable by the designed control scheme (8). Figure 4 and 5 respectively, present the evolution of $\dot{\delta}_{1}\left(x_{1}(t)\right)$ and the stabilizing controller. From Figure 4, we observe that the obtained control gain matrices are switched between the constraints $\mathbb{B}_{1}$ and $\mathbb{B}_{2}$ based on $\dot{\delta}_{1}\left(x_{1}(t)\right)<0$ and $\dot{\delta}_{1}\left(x_{1}(t)\right) \geq 0$, respectively.

According to the structure of conventional SDC in (30), by using the same aforesaid parameter values in Corollary 1, the LUB of sampling interval is calculated as $\bar{d}=0.1090$ and $\bar{d}=0.1140$ for system (36) based on the constraints $\mathbb{B}_{1}:\left\{\widetilde{P}_{1}>\widetilde{P}_{2}, \widetilde{R}_{1}>\widetilde{R}_{2}, \widetilde{W}_{1}>\widetilde{W}_{2}, \widetilde{Q}_{1}>\widetilde{Q}_{2}\right\}$, and $\mathbb{B}_{2}:\left\{\widetilde{P}_{1} \leq \widetilde{P}_{2}, \widetilde{R}_{1} \leq \widetilde{R}_{2}, \widetilde{W}_{1} \leq \widetilde{W}_{2}, \widetilde{Q}_{1} \leq \widetilde{Q}_{2}\right\}$, respectively. It is clear that $\bar{d}=0.1090$ is the final LUB of sampling interval for (36) by the conventional SDC (30). To show the superiority of the obtained results, the comparison of LUB of sampling interval by different methods with Corollary 1 is tabulated in Table 1 .

TABLE 1. LUB of sampling interval $\bar{d}$ in Example 1

\begin{tabular}{|c|c|c|c|c|}
\hline Method & {$[28$} & {$[13$} & {$[14$} & Corollary $[1$ \\
\hline$d$ & 0.0016 & 0.0022 & 0.0253 & 0.1090 \\
\hline
\end{tabular}

As seen in Table 1, the obtained LUB of sampling interval in Corollary 1 is larger than the various methods in [13, 14, 28], which indicates the advantages of proposed theoretical results. 
Fuzzy membership function dependent switched control for nonlinear systems with memory sampled-data information 19

Example 2. The nonlinear mass-spring system [32] is considered as follows:

$$
M \ddot{y}(t)+g(y(t))=\Phi(\dot{y}(t)) u(t)
$$

where $M$ represents the mass, $u(t)$ denotes the control force, and $y(t)$ is the position vector of the spring. $g(y(t))$ and $\Phi(\dot{y}(t))$ are the nonlinear terms of spring and input, respectively.

Assume that $y(t) \in[-a, a]$ and $\dot{y}(t) \in[-b, b]$. Let $\Phi(\dot{y}(t))=1, g(y(t))=a_{1} y(t)+a_{2} y^{3}(t)$ and define $x_{1}(t)=y(t), x_{2}(t)=\dot{y}(t)$, which yields the following system:

$$
\left\{\begin{array}{l}
\dot{x}_{1}(t)=x_{2}(t) \\
\dot{x}_{2}(t)=-\frac{a_{1}}{M} x_{1}(t)-\frac{a_{2}}{M} x_{2}^{3}(t)+\frac{1}{M} u(t) .
\end{array}\right.
$$

Also, the system (38) can be written as T-S fuzzy model (9), where

$$
A_{1}=\left[\begin{array}{cc}
0 & 1 \\
-\frac{a_{1}}{M}-\frac{a_{2}}{M} a^{2} & 0
\end{array}\right], A_{2}=\left[\begin{array}{cc}
0 & 1 \\
-\frac{a_{1}}{M} & 0
\end{array}\right], B_{1}=B_{2}=\left[\begin{array}{c}
0 \\
\frac{1}{M}
\end{array}\right]
$$

with $M=0.97, a_{1}=1.98, a_{2}=1.51$, and fuzzy membership functions $\delta_{1}\left(x_{1}(t)\right)=\frac{x_{1}^{2}(t)}{a^{2}}$ and $\delta_{2}\left(x_{1}(t)\right)=1-\delta_{1}\left(x_{1}(t)\right)$. Based on the switching rules (6), we have the following constraints

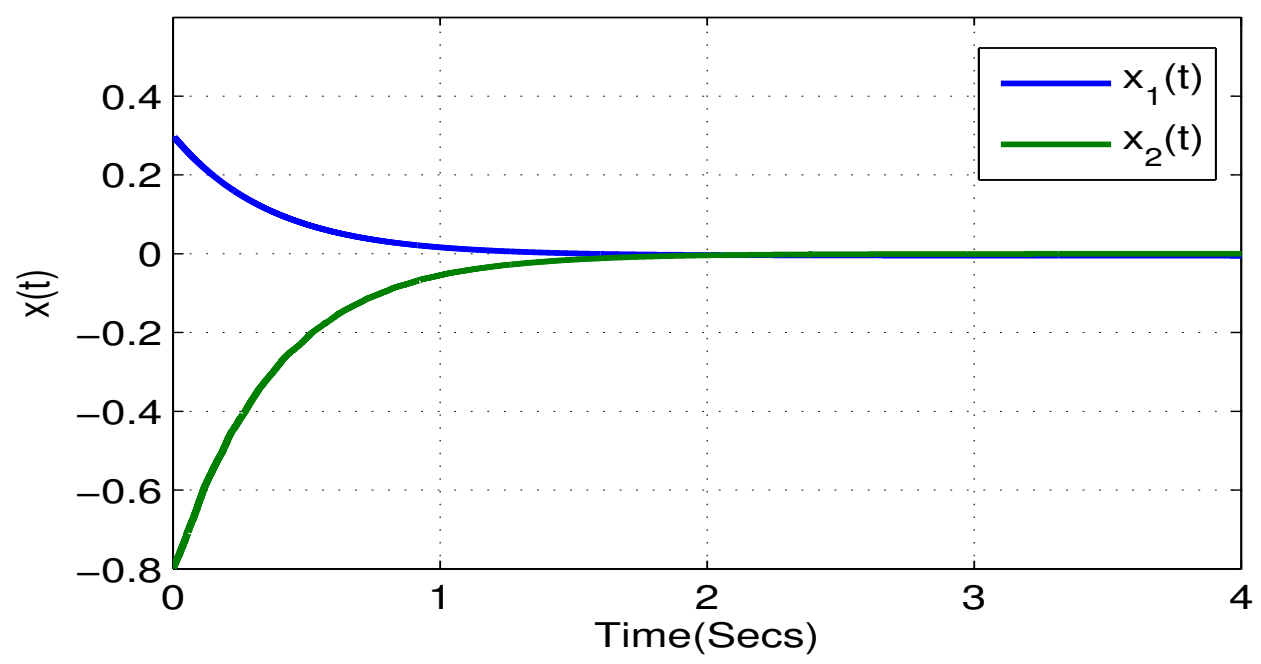

FiguRE 6. State trajectories of the system 38.

$$
\mathbb{B}_{1}:\left\{\widetilde{P}_{1}>\widetilde{P}_{2}, \widetilde{U}_{1}>\widetilde{U}_{2}, \widetilde{W}_{1}>\widetilde{W}_{2}, \widetilde{Q}_{1}>\widetilde{Q}_{2}, \widetilde{S}_{1}>\widetilde{S}_{2}, \widetilde{R}_{1}>\widetilde{R}_{2}\right\}
$$




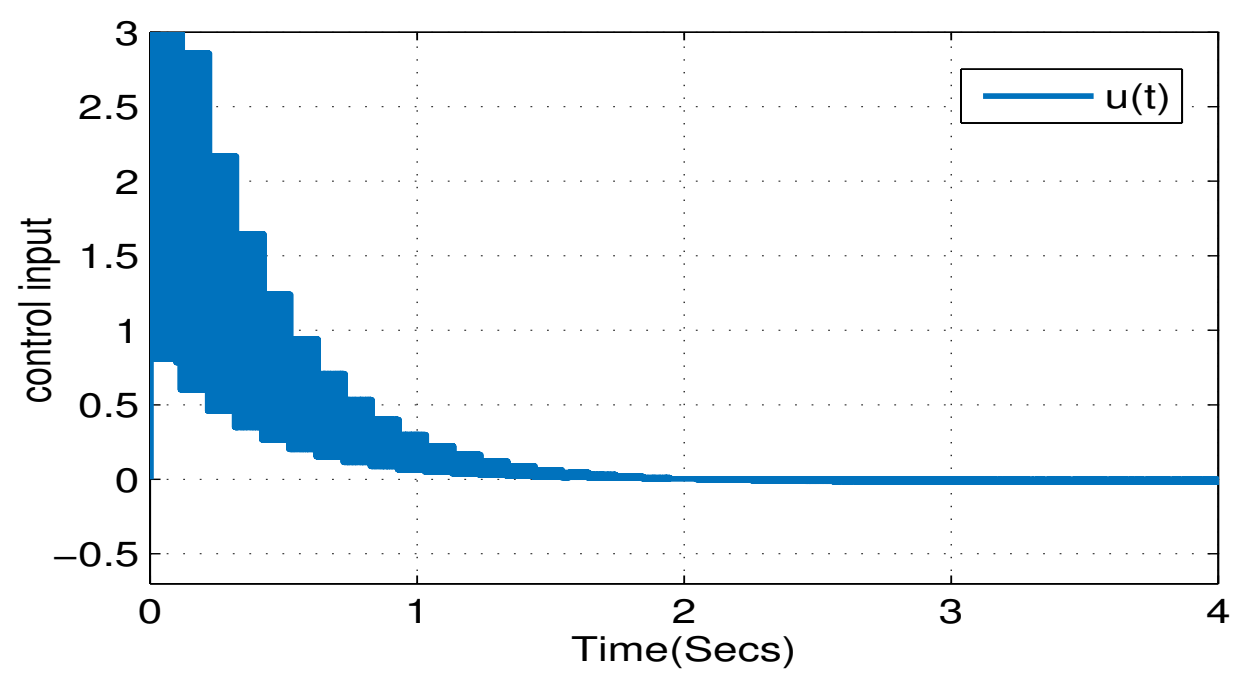

FiguRE 7. Responses of $u(t)$ in Example 2 .

$$
\mathbb{B}_{2}:\left\{\widetilde{P}_{1} \leq \widetilde{P}_{2}, \widetilde{U}_{1} \leq \widetilde{U}_{2}, \widetilde{W}_{1} \leq \widetilde{W}_{2}, \widetilde{Q}_{1} \leq \widetilde{Q}_{2}, \widetilde{S}_{1} \leq \widetilde{S}_{2}, \widetilde{R}_{1} \leq \widetilde{R}_{2}\right\}
$$

Now, let us assume that $a=1, b=0.8, \tau=0.02, \lambda_{1}=\lambda_{2}=1$, and $\beta=0.5$, the LUB of sampling interval is calculated as $\bar{d}=0.1072$ by solving $(26)-(29)$ with the constraints $\mathbb{B}_{1}$, and the gain matrices are obtained as follows:

$$
\begin{aligned}
K_{1,1} & =\left[\begin{array}{ll}
0.3740 & -0.8070
\end{array}\right], K_{1,2}=\left[\begin{array}{ll}
0.3314 & -0.8930
\end{array}\right], \\
L_{1,1} & =\left[\begin{array}{ll}
4.5717 & -3.0836
\end{array}\right], L_{1,2}=\left[\begin{array}{ll}
4.5997 & -2.9810
\end{array}\right],
\end{aligned}
$$

Solving 260 - 29 with the constraints $\mathbb{B}_{2}$, we get the LUB of sampling interval $\bar{d}=0.1016$. Also, the following control gain matrices are determined:

$$
\begin{aligned}
K_{2,1} & =\left[\begin{array}{ll}
0.3128 & -0.8774
\end{array}\right], K_{2,2}=\left[\begin{array}{ll}
0.2926 & -0.9864
\end{array}\right], \\
L_{2,1} & =\left[\begin{array}{ll}
3.9423 & -3.0566
\end{array}\right], L_{2,2}=\left[\begin{array}{ll}
3.9072 & -3.1546
\end{array}\right] .
\end{aligned}
$$

Hence, the final LUB of sampling interval $\bar{d}=0.1016$ is derived from Theorem 2 Further, in Figure 6 and 7 respectively, the state trajectories of system (38) and stabilizing controller are plotted based on the control gain values. Figure 6 shows that the trajectories of mass-spring system is asymptotically stable by the memory-based coupling SDC 8), which implies that the proposed method is applicable and effective. 
Fuzzy membership function dependent switched control for nonlinear systems with memory sampled-data information 21

\section{Conclusion}

In this work, the stability and stabilization analysis for nonlinear systems through the fuzzy memory-based coupling SDC has been investigated. Under the consideration of signal transmission delay, Bernoulli sequence and switching approach, a fuzzy memory-based coupling SDC has been designed. To do this, the LKF has been constructed with a fuzzy membership function, and utilized the generalized $N$-order free-matrix inequality, the adequate conditions have been established in terms of LMIs for guaranteeing the stability of T-S fuzzy systems with less conservativeness. In comparison with the existing studies, the LUB of sampling interval has been calculated by the proposed method. At last, the simulation results for the nonlinear system have been demonstrated the merits of presented theoretical results.

\section{DeClarations}

Conflict of Interest The authors declare that they have no conflict of interest.

\section{REFERENCES}

[1] W. M. Haddad and V. Chellaboina, Nonlinear dynamical systems and control: a Lyapunov-based approach, Princeton University Press, (2011).

[2] R. E. Precup, M. L. Tomescu, and C. A. Dragos, Stabilization of Rössler chaotic dynamical system using fuzzy logic control algorithm, International Journal of General Systems, 43 (5) (2014) 413-433.

[3] M. S. Aslam and Q. Li, Quantized dissipative filter design for Markovian switch T-S fuzzy systems with time-varying delays, Soft Computing, 23 (21) (2019) 11313-11329.

[4] H. Shen, M. Xing, Z. G. Wu, and J. H. Park, Fault-tolerant control for fuzzy switched singular systems with persistent dwell-time subject to actuator fault, Fuzzy Sets and Systems, 392 (2020) 60-76.

[5] J. Luo, M. Li, X. Liu, W. Tian, S. Zhong, and K. Shi, Stabilization analysis for fuzzy systems with a switched sampled-data control, Journal of the Franklin Institute, 357 (1) (2020) 39-58.

[6] A. A. Kalat, A robust direct adaptive fuzzy control for a class of uncertain nonlinear MIMO systems, Soft Computing, 23 (19) (2019) 9747-9759.

[7] X. Zhao, C. Lin, B. Chen, and Q. G. Wang, A novel Lyapunov-Krasovskii functional approach to stability and stabilization for T-S fuzzy systems with time delay, Neurocomputing, 313 (2018) 288-294.

[8] H. Mkaouar and O. Boubaker, Chaos synchronization for master slave piecewise linear systems: application to Chua's circuit, Communications in Nonlinear Science and Numerical Simulation, 17 (3) (2012) 1292-1302.

[9] R. Sakthivel, K. Boomipalagan, M. A. Yong-Ki, and M. Muslim, Sampled-data reliable stabilization of T-S fuzzy systems and its application, Complexity, 21 (S2) (2016) 518-529. 
[10] Z. Chen, J. Wang, K. Ma, P. Zhu, B. He, and C. Zhang, Novel fuzzy event-triggered adaptive control for nonlinear systems with input hysteresis, Soft Computing, 25 (8) (2021) 6619-6631.

[11] Y. Zhang, Robust stability and $H_{\infty}$ control of discrete-time uncertain impulsive systems with time-varying delay, Circuits, Systems, and Signal Processing, 35 (11) (2016) 3882-3912.

[12] H. Shen, L. Su, and J. H. Park, Reliable mixed $H_{\infty} /$ passive control for T-S fuzzy delayed systems based on a semi-Markov jump model approach, Fuzzy Sets and Systems, 314 (2017) 79-98.

[13] Z. P. Wang and H. N. Wu, On fuzzy sampled-data control of chaotic systems via a time-dependent Lyapunov functional approach, IEEE Transactions on Cybernetics, 45 (4) (2014) 819-829.

[14] Y. Wang, Y. Xia, and P. Zhou, Fuzzy-model-based sampled-data control of chaotic systems: a fuzzy time-dependent Lyapunov-Krasovskii functional approach, IEEE Transactions on Fuzzy Systems, 25 (6) (2016) 1672-1684.

[15] Z. Qu, Z. Zhang, Z. Du, and T. Zhao, Fuzzy optimal control for nonlinear systems with time-varying delay via sampled-data controller, Soft Computing, 24 (19) (2020) 14743-14755.

[16] F. Yang, H. Zhang, and Y. Wang, An enhanced input-delay approach to sampled-data stabilization of T-S fuzzy systems via mixed convex combination, Nonlinear Dynamics, 75 (3) (2014) 501-512.

[17] J. Yoneyama, Robust sampled-data stabilization of uncertain fuzzy systems via input delay approach, Information Sciences, 198 (2012) 169-176.

[18] M. S. Ali, R. Vadivel, A. Alsaedi, and B. Ahmad, Extended dissipativity and event-triggered synchronization for T-S fuzzy Markovian jumping delayed stochastic neural networks with leakage delays via fault-tolerant control, Soft Computing, 24 (5) (2020) 3675-3694.

[19] H. B. Zeng, K. L. Teo, Y. He, and W. Wang, Sampled-data-based dissipative control of TS fuzzy systems, Applied Mathematical Modelling, 65 (2019) 415-427.

[20] Y. Tian and Z. Wang, Extended dissipativity analysis of singular Takagi-Sugeno fuzzy systems with time delay via two improved techniques, International Journal of Systems Science, 51 (11) (2020) 2068-2078.

[21] H. B. Zeng, K. L. Teo, Y. He, and W. Wang, Sampled-data stabilization of chaotic systems based on a TS fuzzy model, Information Sciences, 483 (2019) 262-272.

[22] R. Zhang, D. Zeng, J. H. Park, Y. Liu, and S. Zhong, A new approach to stabilization of chaotic systems with nonfragile fuzzy proportional retarded sampled-data control, IEEE Transactions on Cybernetics, 49 (9) (2018) 3218-3229.

[23] C. Ge, Y. Shi, J. H. Park, and C. Hua, Robust $H_{\infty}$ stabilization for TS fuzzy systems with time-varying delays and memory sampled-data control, Applied Mathematics and Computation, 346 (2019) 500-512.

[24] C. Ge, J. H. Park, C. Hua, and X. Guan, Dissipativity analysis for TS fuzzy system under memory sampled-data control, IEEE Transactions on Cybernetics, 51 (2) (2019) 961-969.

[25] P. H. Coutinho, R. F. Araujo, A. T. Nguyen, and R. M. Palhares, A multiple-parameterization approach for local stabilization of constrained Takagi-Sugeno fuzzy systems with nonlinear consequents, Information Sciences, 506 (2020) 295-307.

[26] T. H. Lee and J. H. Park, New methods of fuzzy sampled-data control for stabilization of chaotic systems, IEEE Transactions on Systems, Man, and Cybernetics: Systems, 48 (12) (2017) 2026-2034. 
Fuzzy membership function dependent switched control for nonlinear systems with memory sampled-data information 23

[27] Y. Xia, J. Wang, B. Meng, and X. Chen, Further results on fuzzy sampled-data stabilization of chaotic nonlinear systems, Applied Mathematics and Computation, 379 (2020) 125225.

[28] Z. G. Wu, P. Shi, H. Su, and J. Chu, Sampled-data fuzzy control of chaotic systems based on a T-S fuzzy model, IEEE Transactions on Fuzzy Systems, 22 (1) (2013) 153-163.

[29] A. Seuret, A novel stability analysis of linear systems under asynchronous samplings, Automatica, 48 (1) (2012) $177-182$.

[30] C. Hua, S. Wu, and X. Guan, Stabilization of TS fuzzy system with time delay under sampled-data control using a new looped-functional, IEEE Transactions on Fuzzy Systems, 28 (2) (2019) 400-407.

[31] P. Tang and Y. Ma, Exponential stabilization and sampled-date $H_{\infty}$ control for uncertain T-S fuzzy systems with time-varying delay, Journal of the Franklin Institute, 356 (9) (2019) 4859-4887.

[32] J. Zhao, S. Xu, and J. H. Park, Improved criteria for the stabilization of TS fuzzy systems with actuator failures via a sampled-data fuzzy controller, Fuzzy Sets and Systems, 392 (2020) 154-169.

[33] L. Wang, J. Liu, and H. K. Lam, Further study on stabilization for continuous-time Takagi-Sugeno fuzzy systems with time delay, IEEE Transactions on Cybernetics, (2020).

[34] S. Kuppusamy and Y. H. Joo, Non-fragile retarded sampled-data switched control of TS fuzzy systems and its applications, IEEE Transactions on Fuzzy Systems, 28 (10) (2019) 2523-2532.

[35] H. B. Zeng, X. G. Liu, W. Wang, and S. P. Xiao, New results on stability analysis of systems with time-varying delays using a generalized free-matrix-based inequality, Journal of the Franklin Institute, 356 (13) (2019) 7312-7321. 